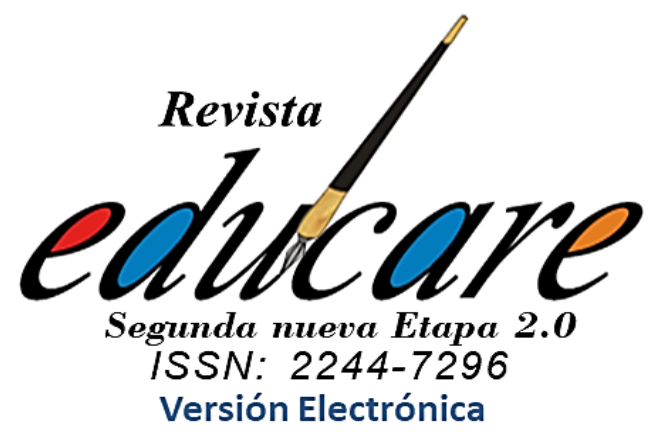

Volumen 24 № 2 Mayo-Agosto 2020

(190-211)

Fabio Fidel Mendoza*

ORCID: https://orcid.org/0000-0002-5155-7752

*Doctorando en Educación - UPEL. MSc. En Informática Educativa - URBE. Experto en Medios y Periodismo Digital - FATLA. Experto en Educación Virtual - FATLA. Esp. En Administración de la Informática Educativa UDES. Lic. En Informática Educativa y Medios Audiovisuales - UNICORDOBA. Correo electrónico fafimego@gmail.com / fafimego@hotmail.com

\section{MEDIACIÓN TECNOLÓGICA ORIENTADA AL DESARROLLO DE HABILIDADES COGNITIVAS: APORTES PARA LA SOCIEDAD DEL CONOCIMIENTO}

TECHNOLOGICAL MEDIATION ORIENTED TO THE DEVELOPMENT OF COGNITIVE SKILLS: CONTRIBUTIONS TO THE KNOWLEDGE SOCIETY

\section{Recibido: \\ 16-03-2019 \\ Aceptado: \\ 13-07-2020}



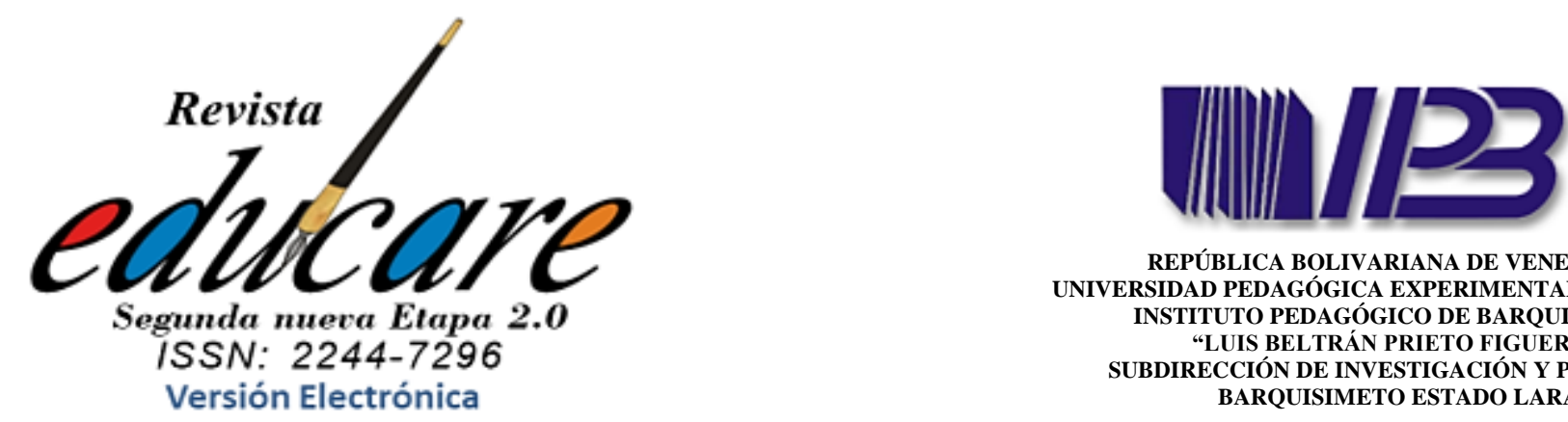

REPÚBLICA BOLIVARIANA DE VENEZUELA UNIVERSIDAD PEDAGÓGICA EXPERIMENTAL LIBERTADOR INSTITUTO PEDAGÓGICO DE BARQUISIMETO "LUIS BELTRÁN PRIETO FIGUEROA

SUBDIRECCIÓN DE INVESTIGACIÓN Y POSGRADO BARQUISIMETO ESTADO LARA

Volumen 24 № 2 Mayo-Agosto 2020

$(190-211)$

\section{Resumen}

Los acelerados avances tecnológicos obligan a los sistemas educativos del mundo a gestionar una sostenida dinámica evolutiva en sus procesos. Expertos en el tema señalan que la clave está en aprender a aprovechar el potencial organizativo, didáctico de las Tecnologías de la Información y Comunicación para mediar aprendizajes en la era informacional. El mismo se sustentó en los aportes teóricos de: SENA (2019), PISA (2018), Pérez, Builes y Rivera (2017), Valencia (2016), Tobón (2013), entre otros. El presente artículo analizó los aportes que genera la mediación tecnológica a la sociedad del conocimiento, orientada al desarrollo de habilidades cognitivas. Por tanto, es un estudio de tipo documental, que utiliza como técnica la recolección de información que da base al análisis de contenido, este implica acciones analíticas y reflexivas en relación a la temática. Se concluye que la mediación tecnológica implica un conjunto de acciones de orden pedagógico, didáctico, organizativo y comunicacional que permiten sacar provecho a los recursos tecnológicos para que los estudiantes adquieran un estilo de aprendizaje independiente para autoformarse con criterios de pertinencia y calidad.

Palabras clave: Cognición, mediación, habilidades cognitiva, sociedad,

TECHNOLOGICAL MEDIATION

ORIENTED TO THE DEVELOPMENT

OF COGNITIVE SKILLS:

CONTRIBUTIONS TO THE

KNOWLEDGE SOCIETY tecnología.

\section{Abstract}

The accelerated technological advances force the educational systems of the world to manage a sustained evolutionary dynamics in their processes. Experts on the subject point out that the key is to learn to take advantage of the organizational, didactic potential of Information and Communication Technologies to mediate learning in the information age. It was based on the theoretical contributions of: SENA (2019), PISA (2018), Pérez, Builes and Rivera (2017), Valencia (2016), Tobón (2013), among others. This article analyzed the contributions generated by technological mediation to the knowledge society, aimed at the development of cognitive skills. Therefore, it is a documentary-type study, which uses the collection of information as a technique that provides the basis for content analysis; this involves analytical and reflective actions in relation to the subject. It is concluded that technological mediation implies a set of pedagogical, didactic, organizational and communicational actions that allow taking advantage of technological resources so that students acquire an independent learning style to self-train with criteria of relevance and quality.

Keywords: Cognition, mediation, cognitive skills, society, technology. 


\section{Introducción}

En la actualidad la humanidad ha sido testigo de los sorprendentes cambios y transformaciones que constantemente se experimentan en el orden, político, económico y social producto de los acelerados avances tecnológicos que hacen que los seres humanos enfrenten a diario el desafío de evolucionar constantemente. Ello demanda una transformación en el ámbito educativo que obliga a docentes, estudiantes y directivos a ser conscientes del momento histórico que les ha tocado vivir y, en consecuencia, se preocupen por establecer estrategias que permitan desarrollar la capacidad de aprender de manera independiente mediante la tecnologías de la información y la comunicación TIC, poniendo en práctica la creatividad para innovar y reflexionar constantemente sobre los procesos educativos que requiere la humanidad.

Así, entre los beneficios que aporta la incorporación de las TIC en los procesos educativos al servicio de la sociedad del conocimiento, destaca sin duda alguna la diversidad de recursos y herramientas didácticas disponibles para estimular el desarrollo del pensamiento lógico y la innovación en el contexto de un aprendizaje autónomo (autodidacta). En efecto, estos recursos representan una opción de ejercitar permanentemente procesos cognitivos de atención, descripción, comparación, análisis y síntesis; y en esa dinámica, se fortalecen las capacidades humanas asociadas a habilidades cognitivas de atención, concentración, memoria y comprensión; todas estas fundamentales para entender un mundo globalizado y adaptarse a los cambios que en él a diario subyacen.

No obstante, aprovechar el potencial cognitivo de las TIC representa un reto complejo que pasa por formar a los docentes con el compromiso y la capacitación necesaria para que asuman el rol de mediadores que demanda la educación del futuro; una vez comprometidos estos con la mediación tecnológica, se requiere avanzar integrando estas herramientas a los procesos formativos para que sus beneficios puedan ser accesibles a todos los actores de la educación. Así lo asume la UNESCO (2013), cuando establece que:

El potencial que las TIC ofrecen para representar y transmitir información no representa en sí mismo un aporte a los procesos de enseñanza y aprendizaje, sino que depende de la apropiación que el docente haga de ellas al integrarlas al sistema simbólico, que puede estar presente en cualquier tipo de escenario educativo (lengua oral, escrita, 
lenguaje, audiovisual, gráfico, numérico, estético, entre otros), en pro de la creación de condiciones inéditas relacionadas con los objetivos educativos que se haya propuesto. (p. 11)

De manera que, una mediación tecnológica centrada en el desarrollo de habilidades cognitivas requiere estar fundamentada en sólidos valores de constancia, disciplina y compromiso; tales valores abrirán paso a la reflexión sobre la importancia de formar a las futuras generaciones como personas auto - didactas; capaces de discernir sobre la veracidad y pertinencia de la abundante información que a diario circula por la internet; y en ese sentido, desarrollar el pensamiento crítico como un indicador importante para consolidar la educación de calidad que demanda la sociedad del conocimiento.

A nivel mundial, existen estudios que demuestran que una gran cantidad de estudiantes no tienen desarrolladas las habilidades del pensamiento. Uno de ellos es el informe dado en Bruselas (Bélgica), en el 2009 y publicado en 2010 sobre "Las habilidades y competencias del siglo XXI para los aprendices del nuevo milenio en los países de la Organización para la Cooperación y el Desarrollo Económico (OCDE)". Según dicho informe, estos países deben identificar tanto las competencias, como las habilidades necesarias de cara a los estándares educativos que cada estudiante debe tener al finalizar su bachillerato o secundaria (OCDE, 2010).

Desde esta perspectiva, la realidad Latinoamérica comparte la situación antes descrita, revelando indicadores negativos que la posicionan por debajo de lo esperado, tal como se demuestra en las pruebas realizadas por el Programa de Evaluación Internacional de Estudiantes PISA (2018), que los países latinoamericanos, se ubicaron en el tercio más bajo del ranking en el desarrollo de habilidades del pensamiento. En efecto, de 65 sistemas educativos evaluados, los países latinoamericanos obtuvieron un rendimiento bajo al resto de las naciones analizadas. De manera que, todavía existe camino por recorrer para que las naciones latinoamericanas logren nivelarse con las habilidades y competencias exigidas por el mercado laboral global que, fundamentalmente, demanda habilidades cognitivas socioemocionales o comportamentales como responsabilidad, trabajo en equipo, pensamiento crítico y la habilidad para resolver problemas. 
Por su parte, Colombia se incluye en esta situación, en efecto, a expresión del Banco Mundial (2018, p. 23), la educación se encuentra lejos de responder a las realidades y necesidades del país, puesto que, a pesar de que se proporcionaron computadoras para las aulas los dispositivos no estaban integrados de forma adecuada en los planes de estudio, por ello, no tuvo impacto en el aprendizaje. Esta realidad, en el contexto colombiano también es reseñada por un estudio del SENA (2019), el cual expresa que hace varios años atrás, el modelo de educación tradicional no enseñó a los educandos a pensar, tampoco a ser críticos y reflexivos. Por ende, reciben como legado de este tipo de modelo, costumbres de abstención intelectual, los cuales lo hacen impasibles.

De esa forma, para contrarrestar este modelo tradicional algunos docentes e investigadores de la pedagogía han propuesto la opción de un aprendizaje activo y significativo, el cual conduzca a una enseñanza centrada en el desarrollo del pensamiento sin dejar a un lado la integración de las TIC en dicho proceso. Mientras, sobre la base de los múltiples diagnósticos como los de Causado, Santos y Calderón (2015) que, se plantearon como objetivo describir el desarrollo del pensamiento crítico en el área de ciencias naturales de una escuela secundaria, se puede esgrimir que, los estudiantes y profesores están en los primeros niveles para desarrollar esta competencia, además, poseen problemas de pensamiento y no tienen claro como pensar de manera crítica, aunado a eso, los docentes no saben cómo intervenir de forma pedagógica para desarrollarlo.

Ello, de cara a los postulados de Dewey (1989), permite inferir que la habilidad de pensar en las consecuencias de un acto se encuentra limitada, en efecto, según el mencionado autor, a muchas personas la búsqueda intelectual y los eventos que exigen razonamiento para actuar les producen malestar, lo cual les lleva a actuar de manera impulsiva; de allí las manifestaciones de agresividad e impulsividad de situaciones difíciles que ameritan un pensamiento reflexivo.

De manera que, varios docentes colombianos, a diario observan con preocupación la agresividad frecuente de sus estudiantes producto de la impulsividad, tanto entre sus pares, como de parte de éstos hacia sus docentes. Situación similar se refleja en las actividades para análisis de texto, donde les cuesta asumir el reto de analizar y realizar inferencias y opiniones para construir uno nuevo. Asimismo, sus habilidades del pensamiento se perciben limitadas, y en varios casos ausentes. 
Por otra parte, en el escenario descrito se observa que los recursos tecnológicos son poco aprovechados en los procesos de mediación. En efecto, la resistencia al uso de la tecnologías por parte de los docentes en sus labores cotidianas, de allí se infiere que existen carencias asociadas a su capacitación para el uso efectivo de estos recursos que, a la vez, pudieran estar afectando la focalización de la diversidad cognitiva generando falencias en la atención, mediación de procesos cognitivos como atención - concentración, memoria y comprensión.

De continuar esta situación, directivos y docentes de las instituciones educativas de Sincelejo permanecerán cerrados a la posibilidad de considerar nuevos estilos de aprendizaje que puedan ser utilizados para coadyuvar en la mejora de tales deficiencias mediante el desarrollo de habilidades del pensamiento a través del uso de herramientas tecnológicas, como estrategia educativa, para el mejoramiento del proceso de enseñanza y aprendizaje.

Las situaciones que se mencionaron anteriormente, invitan a reflexionar sobre los diferentes modelos de mediación tecnológica para el desarrollo de las habilidades cognitivas, con el fin de potenciar las habilidades del pensamiento en los estudiantes y contribuir en el proceso de aprendizaje, adicionalmente, esto conlleva un conocimiento para la vida sobre las TIC que responde a las exigencias y demandas de la sociedad del conocimiento

Así, lo planteado conduce a la formulación de la siguiente interrogante de investigación: ¿Cuáles elementos conformarán el modelo de mediación tecnológica centrado en el desarrollo de habilidades cognitivas para atender la demanda de la sociedad del conocimiento?

De esta interrogante central derivan otras sub - interrogantes que contribuyen a la comprensión del problema; a continuación se presentan:

¿Cómo deben ser aprovechados los recursos tecnológicos para la mediación que exige la sociedad del conocimiento?

¿Cómo diagnosticar la diversidad cognitiva en cuanto al nivel de desarrollo de las habilidades cognitivas de atención, memorización y comprensión en los estudiantes?

A todos estos cuestionamientos busca responder el presente estudio documental en aras de ampliar el acervo científico sobre el tema. En ese sentido, se propone analizar los aportes que genera la mediación tecnológica orientada al desarrollo de habilidades cognitivas: Aportes para la sociedad del conocimiento. 
De allí que para llevar a cabo se conduce este estudio hacia una investigación de tipo documental, la cual según Hernández, Fernández y Baptista (2014), se hace énfasis en la reflexión crítica sobre la mediación tecnológica orientada al desarrollo de habilidades cognitivas. Además, la recolección de información que da asiento a esta investigación es el análisis de contenido, este implica acciones analíticas y reflexivas en relación a la temática, en las cuales se verifican las teorías existentes y las investigaciones previas (Hurtado, 2015). El abordaje de la temática se realiza mediante los apartados discursivos referidos que hablan sobre el aprovechamiento de los recursos tecnológicos y el diagnóstico de la diversidad para determinar el nivel de desarrollo de las habilidades cognitivas.

Para ello, en primer lugar se ofrecen orientaciones acerca de cómo aprovechar los recursos tecnológicos para la mediación que exige la sociedad del conocimiento. Seguidamente, se dan a conocer algunos aportes científicos que orientan en torno al diagnóstico de la diversidad cognitiva para determinar nivel de desarrollo de las habilidades cognitivas de atención, memorización y comprensión en los estudiantes.

Asimismo, se dan a conocer algunas consideraciones sobre las actividades para el desarrollo de las habilidades cognitivas de atención, memorización y comprensión en el marco de una mediación tecnológica. Finalmente, sobre la base de estas consideraciones se presentan algunas reflexiones finales acerca de los elementos que deben considerarse en un modelo de mediación tecnológica centrado en el desarrollo de habilidades cognitivas como aporte a la demanda pedagógica de la sociedad del conocimiento.

\section{¿Cómo aprovechar los recursos tecnológicos para la mediación que exige la sociedad del conocimiento?}

El aprovechamiento de los recursos tecnológicos constituye una acción planificada que necesariamente debe estar enmarcada en un proyecto educativo con propósitos bien definidos que garantice el uso efectivo de las tecnologías de conformidad con los resultados esperados. Aunado a ello, dicho proyecto debe también garantizar una formación docente acorde a los retos educativos subyacentes.

En ese orden de ideas, Pérez, Builes y Rivera (2017), lograr la integración de las TIC en el aula dependerá de la efectividad de la apropiación tecnológica y de las estrategias de enseñanza, es decir, de la capacidad de los docentes para estructurar un ambiente de 
aprendizaje no tradicional que permita fusionar las TIC con nuevas didácticas; fomentar clases dinámicas en el plano social, estimular la interacción cooperativa, el aprendizaje colaborativo y el trabajo en equipo.

En ese sentido, la introducción de las TIC en la mediación destaca la necesidad de redefinir los roles de docentes y estudiantes en los contextos educativos. En relación a los estudiantes, gracias a estas nuevas herramientas pueden adquirir mayor autonomía y responsabilidad en el proceso de aprendizaje, ello obliga al docente a salir de su rol clásico como única fuente de conocimiento (UNESCO, 2013). Entonces, el éxito en cuanto al provecho de los recursos tecnológicos para la mediación está determinado por diversos factores entre los que destaca la manera en que ellos se integran las TIC a los procesos educativos.

En comunión con la postura anterior Valencia y otros $(2016$, p. 8) destacan que la expectativa benéfica de las TIC en el sistema educativo y las condiciones en las que dicha expectativa se hace posible expresan "la necesidad de realizar cambios en todas sus áreas (técnica, pedagógica, administrativa, directiva)" (p.8) para que se puedan producir experiencias educativas eficaces y efectivas que "favorezcan los procesos de enseñanza y aprendizaje" (p.12); en ese sentido, es importante que las autoridades educativas se acojan a modelos de formación que les permitan adaptarse a los cambios permanentes que hace parte de una sociedad de la información y el conocimiento. De manera que, el provecho de los recursos tecnológicos para la mediación constituye un aspecto fundamental a considerar para implementar un modelo de mediación tecnológica.

Con relación al aprovechamiento de contenidos digitales, se trata de codificar, almacenar y recuperar cuando sean necesarios los contenidos grabados en formato digital para ponerlos al servicio del sistema educativo. Ello implica la configuración de un proceso para la gestión del conocimiento adaptado a las características, intereses y necesidades de los actores educativos y a su vez, la implementación de una cultura organizacional centrada en valores de cooperación, comunicación y trabajo en equipo que permita por una parte, ser garante de un ejercicio docente efectivo e innovador, y por otra, el desarrollo de procesos y habilidades favorables al desarrollo cognitivo de los estudiantes.

En esa dinámica, según Hernández, Orrego y Quiñones (2018), la capacitación docente constituye un aspecto clave, puesto que brinda a los profesores las herramientas necesarias para utilizar las TIC en la producción de contenidos de cualquier área. Por ende, se pretende 
capacitar al docente para que mediante la interacción cognitiva con sus colegas y estudiantes logren construir el conocimiento aprovechando las bondades de las TIC a la luz de un currículo abierto y flexible.

En efecto, Lugo y Kelly (2011), destacan la importancia de los procesadores de texto, las planillas de cálculo y los programas para el diseño de sitios web, como herramientas que puede aprovechar cada docente para llevar a cabo la producción de contenidos digitales como labor fundamental en la mediación tecnológica. Así, estos recursos brindan la posibilidad de crear contenidos digitales a partir de la sistematización de actividades pedagógicas en cualquier área de estudio, las cuales pueden ser archivadas, publicadas y compartidas con otros actores educativos mediante el internet.

También, existe la posibilidad de soportar o acompañar estos contenidos con imágenes, videos y simuladores, a fin de estimular el potencial de estos programas como herramienta de aprendizaje. Desde ese punto de vista, la producción de contenidos constituye una acción estratégica de aprendizaje colaborativo entre docentes y estudiantes. En este sentido, Lugo y Kelly (2011), afirma que los contenidos, no se presentan directamente, sino que es el aprendiz el que los va creando y construyendo según sus intereses, actitudes y necesidades, y esto se puede conseguir de manera individual o frecuentemente con la colaboración de otros en trabajo colaborativo.

De manera que, las actividades que involucran estrategias de aprendizaje colaborativo provocan entusiasmo en los estudiantes. Por ende, se deduce que es ese entusiasmo el que actúa como elemento motivador en la producción de contenidos digitales, logrando así la efectividad del aprendizaje tanto a nivel individual como a nivel colectivo (Morrissey, 2011). Así, se infiere la importancia de orientar la capacitación docente hacia la consolidación de una cultura de producción científica que estimule la producción de estrategias de aprendizaje colaborativo, la producción escrita de contenidos propios de las diferentes áreas de estudio, a fin de publicarlos y compartirlos con pares académicos.

Con respecto a la dotación de equipos tecnológicos, los equipos representan un recurso fundamental en el que se piensa al momento de dotar la infraestructura tecnológica de una organización educativa, por consecuencia, se trata de aprovechar al máximo los recursos TIC disponibles en la escuela, familia y comunidad para ponerlos al servicio del sistema educativo. Ello pasa por realizar un diagnóstico en cada uno de estos escenarios, para luego elaborar un 
plan de integración acorde a la realidad diagnosticada, a fin de garantizar la efectividad y la pertinencia de estas tecnologías en su contexto de aplicación, pero sobre todo, esta acción facilitará la medición del impacto que causan estas tecnologías en el contexto donde fueron aplicadas.

Así, reafirmando la importancia de proporcionar a las instituciones educativas los recursos TIC necesarios, Morrissey (2011), afirma que estos deben ser confiables, de fácil acceso y sobre todo; deben estar disponibles para ser utilizados por docentes y estudiantes en todo momento. En este sentido, el autor antes citado pone de relieve la idea de ubicar estos recursos al servicio de los procesos educativos sin perder de vista que éstos equipos son solo herramientas que requieren de una mediación adecuada para fortalecer los métodos educativos.

Por su parte, Navarro (2010), coincide con el autor referido al afirma que "son los recursos tecnológicos los que deben girar alrededor del acto educativo y no a la inversa" (p. 9). Vistos desde esa perspectiva, los equipos tecnológicos constituyen una herramienta al servicio de la mediación que favorece el aprendizaje de cualquier contenido en cualquier área de estudio.

En este mismo sentido, la UNESCO (2013), establece que la formación docente debe ser complementada con la dotación de equipos tecnológicos como herramientas para fortalecer la gestión escolar mediante el diseño de materiales educativos, la construcción de propuestas didácticas innovadoras y la construcción de modelos de evaluación pertinentes a la realidad escolar. Finalmente, el aprovechamiento del internet como recurso tecnológico para la mediación consiste en logar capacitar a docentes y estudiantes para utilizar las características de esta herramienta (conectividad, interactividad, multimedialidad entre otras) para desarrollar procesos y habilidades cognitivas que a su vez faciliten el aprendizaje sobre las TIC (uso y manejo de la tecnologías de la información) y con las TIC (contenidos académicos y de cultura general).

Atendiendo estas consideraciones se puede señalar, que la necesidad de interactuar y estar comunicado en un mundo globalizado por la internet, ha ocasionado que el diseño e implementación de páginas y sitios web sea una labor no solo rentable, sino también necesaria para la llamada sociedad del conocimiento, donde la información se concibe como uno de los recursos más importantes para la toma de decisiones e impacta de manera directa el desarrollo económico y social de los pueblos. 
En tal sentido, las páginas y sitios web como recurso al servicio de una sociedad que ha evolucionado, y por lo tanto pasó de industrial a informacional, ofrecen beneficios, bondades y limitaciones que necesariamente deben ser conocidas para valorar su potencial comunicacional. $\mathrm{Su}$ importancia radica en que constituye un recurso potencialmente efectivo y eficiente capaz de proporcionar un sin fin de posibilidades a un costo extremada y relativamente bajo debido a su amplia cobertura comunicacional a nivel mundial.

En ese escenario, subyacen retos y desafíos que la educación requiere asumir para estar a la vanguardia; en efecto, como ya se ha mencionado, el aprovechamiento del internet como recurso tecnológico para la mediación consiste en logar capacitar a docentes y estudiantes para utilizar las características de esta herramienta (conectividad, interactividad, multimedialidad entre otras) para desarrollar procesos y habilidades cognitivas que a su vez faciliten el aprendizaje sobre las TIC (uso y manejo de la tecnologías de la información) y con las TIC (contenidos académicos y de cultura general) (Morrissey, 2011).

Ahora bien, para hacer realidad lo antes planteado Ortiz (S/A) propone que debe existir un proyecto educativo que contemple estos aspectos; donde el uso y aprovechamiento del internet se oriente a la investigación para la elaboración de material de estudio contextualizado según los requerimientos del entorno, el aporte fundamental se expresa en la adecuación pedagógica del contenido digital encontrado para ser utilizado al servicio del hecho educativo.

Entonces, el uso y aprovechamiento de este recurso no se limita a la selección y trascripción de información, sino que, mediante una adecuada mediación que fortalezca los procesos y habilidades cognitivas trasciende hasta lograr el procesamiento de la misma para generar, almacenar y compartir conocimientos (Yanes, 2011). En ese sentido, el aprovechamiento del internet como recurso tecnológico al servicio de la mediación puede lograr que los estudiantes desarrollen habilidades de búsqueda y selección de la información; y al mismo tiempo, fortalece destrezas y habilidades cognitivas para aprender sobre tecnología y con la tecnología.

El diagnóstico de la diversidad: una herramienta para determinar el nivel de desarrollo de las habilidades cognitivas de atención, memorización y comprensión en los estudiantes

Para Arriaga (2015), el diagnóstico educativo se concibe como un proceso de indagación científica, que se asienta en la epistemología, que está compuesto por sujetos o 
instituciones que abarcan la globalidad de su ambiente e incorporan de forma imprescindible una mediación educativa. En este contexto, este proceso se justifica en la necesidad de brindar una atención educativa que "respete las diferencias del ser humano", donde el educador juega un rol fundamental apropiándose de referentes pedagógicos que le permitan concebir las diferencias como algo consustancial a la naturaleza de las personas; y en ese sentido, poder ofrecer una enseñanza rigurosa y más capacitada para responder al reto de la diversidad (MEN, 2013, p. 21).

Lo anterior devela uno de los desafíos más importantes que subyace en la era informacional con el desarrollo e incorporación de las Tecnologías de la Información y Comunicación a la educación, como lo es la necesidad de brindar oportunidades educativas que incorporen estas tecnologías al sistema educativo adoptando criterios de equidad en cuanto a la calidad del servicio escolar brindado. Adicional a lo antes planteado, por Hernández et al. (2018), cuando afirma, “...el docente concibe el uso de las TIC como un reto y en la actualización de estos procesos conlleva a mejorar la práctica educativa" (p. 681). Lo antes planteado constituye una exhortación a crear las condiciones necesarias para que se atiendan las necesidades cognitivas de todas las personas según sus características e intereses mediante el uso de las TIC.

De manera que, el diagnóstico de la diversidad cognitiva en el marco de un modelo de mediación tecnológica centrado en el desarrollo de habilidades cognitivas en instituciones educativas se concibe como un procesos fundamental y estratégico en el que necesariamente debe estar fundamentada la mediación tecnológica para desarrollar la atención, la memoria y la comprensión mediante el uso y aprovechamiento de recursos tecnológicos (Hepp, 2008).

En ese orden de ideas, el diagnóstico de la diversidad cognitiva permitirá detectar el nivel de desarrollo de la cognición de los estudiantes en tres fases o procesos a saber; identificación, comprensión y sistematización (Yanes, 2011). Asimismo, la fase de identificación es la primera en el orden de ejecución al desarrollar un diagnóstico de la diversidad cognitiva, como su nombre lo expresa, tiene como propósito identificar la diversidad cognitiva presente en los estudiantes (estilos de aprendizaje, características, intereses y demás condiciones biopsicosociales) (Hepp, 2008).

Lo antes mencionado, es similar a lo planteado por Bruzzo, Halperin y Lanci (2010), quienes plantean que la evaluación diagnostica es buen inicio para conocer las habilidades y 
destrezas que poseen los estudiantes inclusive los que presentan cuyas necesidades se derivan de su capacidad o sus dificultades del aprendizaje. Además, se debe respetar la atención a la diversidad escolar al considerar el ritmo, estilo, potencialidades e intereses particulares de los educandos. Por lo tanto, varios estudiantes experimentan dificultades de aprendizaje y tienen por lo tanto necesidades educativas especiales en algún momento de su escolarización.

Otra postura científica similar a la anterior la sostiene Pilonieta (2011), quien añade que cuando las funciones cognitivas son deficientes, la mediación debe centrarse en: 1. Detectar la fase en la cual se presenta la dificultad, 2. Identificar las estrategias correspondientes para corregirlas. 3. Determinar el tipo de aprendizaje y de experiencias necesarias para superarlas. Además, en el caso de estudiantes con necesidades educativas especiales, según Pilonieta (2011), existe un enfoque común para su identificación y evaluación en todos los grupos etéreos, por ende, se requiere de una valoración diagnóstica diferenciada según la edad y la etapa de escolaridad, no obstante la estructura principal y los principios orientadores son los mismos en cada momento.

Una vez identificada la diversidad cognitiva la segunda fase en el orden a seguir para el diagnosticar la diversidad cognitiva es la comprensión. Esta es una fase cuya naturaleza investigativa permite indagar para comprender la diversidad identificada en función de formular e implementar las estrategias más efectivas en el proceso de mediación, y así garantizar un servicio educativo pertinente y de calidad. Su propósito está basado en comprender cada uno de los aspectos que conforman la diversidad del grupo focal para brindar un servicio educativo acorde a las características intereses y necesidades del grupo de estudiantes diagnosticado. (Hepp, 2008).

Así, para brindar una atención educativa que respete las diferencias del ser humano "el educador debe apropiarse de referentes pedagógicos que contemplen las diferencias como algo consustancial a la naturaleza de las personas y poder impartir una enseñanza rigurosa y más capacitada para responder al reto de la diversidad" (MEN, 2013, p. 21).

La postura anterior es compartida por Tobón (2013), quien en su enfoque de formación por competencias destaca "la planeación parte de la comprensión de las finalidades de la formación, y determina cómo, dónde, cuándo y con qué medios se van a formar las competencias" (p, 243). Es decir, que el procesos de comprensión de la diversidad forma parte 
de un plan diagnóstico del cual se desprende el resto de los planes educativos que darán respuesta a la diversidad identificada.

En el contexto de la mediación tecnológica, el proceso de comprensión abarca aspectos mucho más amplios, puesto que no sólo se busca comprender la diversidad en orden a la cognición, sino también con respecto al uso y manejo de las TIC, es decir, se busca comprender la diversidad en torno al aprendizaje sobre y con las TIC. Entonces, como lo explica Pérez, Builes y Rivera (2017, p. 6), se trata de reconocer y adoptar desde un punto de vista didáctico, nuevos métodos de trabajo con ayuda de las tecnologías de la información y comunicación, permitiendo de esta manera que los docentes se capaciten y actualicen con el fin de potenciar los procesos de aprendizajes. En consecuencia, es la escuela el escenario ideal para impulsar estos cambios que de seguro van a transformar la educación para que responda a los estándares de calidad que exige la sociedad del conocimiento.

Una vez que se logra comprender la diversidad, la siguiente fase consiste en sistematizar esos hallazgos para que sirvan como fundamento en la formulación e implementación de las estrategias más efectivas en el proceso de mediación. Ello, sin duda alguna favorecerá la prestación de un servicio educativo pertinente y de calidad. Tobón (2013), destacando su importancia indica que este proceso consiste en redactar las contribuciones de todos los expertos y participantes con detalle, así como de las personas que tienen algún rol en el taller; registra informes de acuerdos y finalmente presenta el informe de los acuerdos alcanzados y de los temas en discusión.

Por otra parte, esta acción necesariamente requiere estar enmarcada en un proyecto educativo que busque ser evaluado y al mismo tiempo nutrido con estos procesos. En efecto, para Martelo, Camaño, Gómez, Pinedo y Castaño (2019), la educación es un medio para desarrollar las capacidades de una persona desde diferentes ámbitos como el intelectual y moral. En este sentido, existen dos partes interesadas en la realización de esta actividad, el estudiante y el profesor, quienes desempeñan funciones de aprendizaje y enseñanza.

En este sentido, los proyectos de aprendizaje se convierten en una pieza importante en el proceso debido a que determina el éxito de la enseñanza, por lo cual se han propuesto diferentes herramientas didácticas para abordar la enseñanza desde puntos de vista innovadores y transformacionales lo cual facilitan el desarrollo adecuado de los procesos de integración 
educativa a todo la población sin distinción de condiciones particulares permitiendo atender al estudiantado en la diversidad.

Entonces, la implementación de un modelo de mediación tecnológica centrado en el desarrollo de habilidades cognitivas en instituciones educativas requiere partir de un diagnóstico de la diversidad cognitiva que tomando en cuenta el proyecto educativo de la institución donde pretende ser aplicado tome en cuenta la sistematización como fase final y eje articulador para facilitar una mediación tecnológica pertinente, coherente y de calidad.

\section{Algunas consideraciones sobre el desarrollo de habilidades cognitivas de atención, memorización y comprensión en el marco de una mediación tecnológica}

Las habilidades cognitivas se conciben como un conjunto de operaciones mentales mediante las cuales un sujeto se apropia del conocimiento. Para Felman (2005), la memoria es una función del cerebro y a la vez un fenómeno de la mente que permite al organismo codificar, almacenar y recuperar información del pasado. Como habilidad cognitiva funciona como un gran archivador que puede ir registrando saberes ideas, experiencias, conceptos, sabores, olores y demás experiencias asimiladas por los sentidos.

Asimismo, el autor antes mencionado manifiesta que "las TIC sirven como soporte de procesos cognitivos como la memoria y la meta cognición, ya que estimulan la conexión del estudiante con operaciones de alto nivel” Felman (2005, p.22). La memoria no solo se adiestra en la infancia sino a lo largo de toda la vida. Existen innumerables formas de ejercitarla comprobándose que una de ellas es el uso de las TIC. No obstante, es la escuela y el profesor en particular el encargado de desarrollar en jóvenes y niños capacidades y habilidades cognitivas mediante el uso de estas tecnologías.

Para Paz et al. (2017), la memoria a largo plazo MLP es directa, inmediata, lenta y compleja. En ese sentido, las nuevas experiencias requieren de tiempo y esfuerzo para ser almacenadas. En esa dinámica, la información retenida se estructura de manera que permita su recuperación posterior. La memoria a corto plazo retiene la interpretación inmediata de los acontecimientos que han tenido lugar (últimas palabras de una frase, nombre de una persona, número de teléfono...). Según los autores esta sólo permite la retención de los últimos cinco o seis ítems de un evento inmediato, siendo esta una de las características más significativas de este almacén. 
A diferencia de la memoria a corto plazo, la capacidad y registro sensoriales de la MLP es ilimitada, no obstante constituye un proceso complejo al momento de recuperar la información que en ella se encuentra, puesto que está organizada en una estructura compleja que conecta entre si los acontecimientos y conceptos elaborados por experiencias pasadas. En este sentido, su efectividad está determinada por la aplicación de mecanismos y estrategias mentales relacionadas con el pensamiento y la solución de problemas.

Con relación al desarrollo de la comprensión mediante el uso de las TIC, es importante destacar que según Dewey (1989), existen tres (3) recursos innatos en la formación del pensamiento reflexivo que el docente debe aprovechar usando la tecnología en su rol de orientador; estos son la curiosidad, las sugerencias y el orden. Con relación a la curiosidad, según el precitado autor esta se define como el factor básico que determina la ampliación de la experiencia constituido por la suma de tendencias de todo órgano sensorial y motriz, por lo tanto constituye un factor primordial que necesariamente debe ser aprovechado en la tarea de formar un pensamiento reflexivo.

Asimismo, su desarrollo según Dewey (1989), consta de tres etapas; 1. Manifestación de energía orgánica. 2. El estímulo social y la tercera (3) y última, la etapa intelectual. Con relación a la etapa de manifestación de energía orgánica, se trata de un torrente vital que lleva al niño a descubrir al mundo interesándose por todo; esta primera expresión dista mucho del pensamiento. Una vez superada esta etapa inicial, se abre paso a la etapa de estímulo social cuando la persona descubre que puede apelar a otros para completar su acervo de experiencia. Finalmente, esta segunda etapa termina evolucionando por encima del nivel orgánico y el nivel social hasta alcanzar un nivel intelectual.

Cabe destacar, la importancia de comprender el desarrollo de cada una de ellas hasta alcanzar la madurez intelectual (independencia), a fin de desarrollar procesos de orientación que sean pertinentes en la tarea docente de evitar que la curiosidad se pierda al verse afectada por diversos factores entre los que destacan la indiferencia, el descuido, la superficialidad y el dogmatismo, todos ellos contribuyentes en la pérdida del espíritu de asombro.

Siguiendo con la descripción de los recursos innatos que deben ser aprovechados en la formación del pensamiento reflexivo, a continuación se describe la sugerencia. Esta se define como el estado primitivo y espontáneo de las ideas (Dewey 1989), producto del intercambio de experiencias. La ampliación de estas experiencias primitivas permite al estudiante asociar la 
percepción de objetos observados con otros grabados en su memoria que le sugieren experiencias pasadas.

En un primer momento, esta actividad se manifiesta de manera espontánea, luego se desarrolla de manera controlada cuando se acepta la responsabilidad de utilizar la sugerencia para indagar qué se desprende de ella; es allí donde actúa la introducción del yo como agente y fuente del pensamiento. Asimismo, la sugerencia opera de manera distinta en cada persona haciéndose evidente en dimensiones como rapidez, alcance, variedad y profundidad.

Por último, el orden se define como la secuencia ordenada de sugerencias que conducen a una conclusión como expresión de un pensamiento reflexivo. Este es el tercer y último recurso a aprovechar en la formación de esta forma de pensamiento. Se trata de convertir las sugerencias en pensamiento reflexivo mediante el control y la organización de las ideas, a fin de que tengan la fuerza intelectual y los argumentos suficientes para demostrar la verdad. Entonces, el pensamiento reflexivo implica secuencialidad, continuidad u ordenamiento de las sugerencias; en consecuencia, impacta directamente la acción. En efecto, a menudo una acción ordenada es un indicador de un pensamiento ordenado (Dewey 1989).

Ahora bien, el pensamiento reflexivo no debe ser concebido como una capacidad única y dogmática de pensar, sino como una diversidad de modos en los que las cosas observadas recordadas y leídas evocan sugerencias o ideas pertenecientes a un problema o cuestión que hacen avanzar la mente hacia una conclusión justificable. Para ello, la formación constituye un aspecto fundamental al desarrollar la curiosidad, la sugerencia y los hábitos de indagación y comprobación para desarrollar la sensibilidad y el amor hacia la investigación.

En comunión con lo antes planteado Díaz y Hernández (2010), sugieren como principios motivacionales el desarrollo de actividades de aprendizaje relacionadas con los intereses de los estudiantes, lo cual implica el otorgamiento de libertad para la toma de decisiones y el ejercicio de la autonomía en aras de fomentar la motivación intrínseca para desarrollar las actividades de aprendizaje.

De acuerdo con lo anterior, para formar un pensamiento reflexivo es importante respetar las características, intereses y necesidades individuales del estudiante, puesto que se trata de una formación que necesariamente requiere focalizar la diversidad atendiendo aspectos relacionados con las condiciones genéricas y específicas del entorno que afectan el aprendizaje e influyen de manera negativa en la formación. 
Específicamente se hace referencia a los hábitos mentales ajenos, tales como juicios infundados y anticipados, excesos en la influencia del maestro como mediador (fomenta dependencia cognoscitiva); inclinación a satisfacer otros actores del procesos formativo como padres y maestros en lugar de interesarse por la solución de un problema, la naturaleza de las asignaturas de estudio, entre otras (Dewey 1989).

\section{Reflexiones Finales}

Toda mediación necesita satisfacer los intereses, características y necesidades de la población atendida. Ello implica la implementación de un modelo didáctico centrado en una filosofía pedagógica coherente con el entorno social y sus procesos cotidianos a los cuales necesariamente debe dar respuesta el hecho educativo.

Así, respondiendo a una demanda global de la sociedad del conocimiento surge el uso de las Tecnología de la Información y Comunicación, como una realidad que hace posible la independencia cognitiva; es decir, aprender de manera independiente. Ello, facilita a los sujetos mediados la posibilidad de administrar mejor su tiempo para aprovechar oportunidades de estudio y desempeñarse en otras ocupaciones de orden social y económico. Por otra parte, el uso de las TIC favorece el desarrollo de habilidades para la cooperación e investigación; el desarrollo de procesos y habilidades cognitivas facilitando una efectiva selección y procesamiento de información de interés.

En ese sentido, la mediación tecnológica tiene el reto de satisfacer necesidades propias de la educación asociada a la tecnología educativa en un contexto global. En efecto, contribuye al desarrollo de habilidades de orden cognitivo y social que permiten optimizar el tiempo; ello se debe a que el aprendizaje sobre tecnología a su vez contribuye al desarrollo de habilidades para aprender con la tecnología (Landazábal, 2011).

Por ende, se plantea como una modalidad que considera los intereses y necesidades de los sujetos mediados, proporcionándoles las condiciones para que puedan desarrollar un aprendizaje independiente. Estas condiciones están relacionadas con la aplicación de un modelo de mediación tecnológica que se pueda socializar con los estudiantes y que garantice el uso y provecho de los recursos tecnológicos para llevar a cabo un diagnóstico de la diversidad cognitiva que refleje la realidad en cuanto al desarrollo de los procesos de cognición (atención, 
memoria y comprensión), lo cual redunda en una educación virtual pertinente y de calidad (Heep, 2008).

El modelo de mediación tecnológica basado en el desarrollo de las habilidades cognitivas, puede ser considerado como unas técnicas de orientación que incluye la pedagogía en las cualidades del espacio virtual, incentivando la interacción y el análisis de las ideas aprendidas (Yanes, 2011), con el objetivo de consolidar un proceso de formación que responda a los estándares de calidad que demanda la sociedad del conocimiento.

El mediador garantiza el desarrollo de dichas habilidades en la medida que demuestre destrezas para el uso y provecho de recursos tecnológicos (contenidos educativos digitales, dotación de equipos tecnológicos e internet) que favorecen el desarrollo de actividades de aprendizaje en entornos virtuales; el aprendizaje colaborativo y de investigación, mediante las cuales se logra el autoaprendizaje (Heep, 2008).

De manera que, se trata de formar estudiantes que aprendan de manera independiente, es decir; que aprendan a aprender. En efecto, una de las funciones principales de la mediación tecnológica es sacar provecho a las herramientas que la tecnología ofrece para hacer realidad el hecho educativo. (Yanes, 2011; Martín, 2008). Ahora bien, con relación a los aspectos antes mencionados, se destaca que el uso efectivo de estas herramientas puede ser determinante para desarrollar el compromiso y la motivación que el estudiante necesita en aras de autoformarse y ser competitivo en el mercado global.

Entonces, representan una oportunidad para que los estudiantes logren desarrollar habilidades asociadas al trabajo cooperativo, aprendizaje cooperativo, la investigación; el análisis y procesamiento de información (Landazábal, 2011); todas ellas muy cotizadas en los mercados laborales en la actualidad. Por lo tanto, se busca preparar a niños y jóvenes para que en el futuro se conviertan en profesionales capaces de enfrentar los retos que plantean la economía global y la sociedad del conocimiento.

Así, un modelo de mediación tecnológica centrado en el desarrollo de habilidades cognitivas en instituciones educativas puede ser concebido como un conjunto de acciones de orden pedagógico - didáctico, organizativo y comunicacional que permiten sacar provecho a los recursos tecnológicos para diagnosticar la diversidad cognitiva en función de desarrollar procesos y habilidades de cognición en los estudiantes favorables para adquirir un estilo de aprendizaje independiente que les permita autoformarse con criterios de pertinencia y calidad. 


\section{Referencias}

Arriaga, M. (2015). El diagnóstico educativo: Una importante herramienta para elevar la calidad de la educación en manos de los docentes. Atenas, 3(31), 63-74. https://www.redalyc.org/pdf/4780/478047207007.pdf

Banco Mundial (2018). Informe sobre el desarrollo mundial 2018: Aprender para hacer realidad la promesa de la educación. Cuadernillo del "Panorama general". Oficina Central, Washington, DC: Banco Mundial Washington. https://observatorioeducacion.org/sites/default/files/aprender._hacer_realidad_la_promes a_de_la_educacion.pdf

Bruzzo, M. Halperin, E. y Lanci, C. (2010). Educación Especial. Integración en la Escuela. Argentina: Grupo Clasa.

Causado, R., Santos, B., Calderón, I. (2015). Desarrollo del pensamiento crítico en el área de ciencias naturales en una escuela secundaria. Revista Facultad de Ciencias Universidad $\begin{array}{llll}\text { Nacional } & \text { de } & \text { Colombia, } & \text { 17-42. }\end{array}$ https://revistas.unal.edu.co/index.php/rfc/article/view/51437

Dewey, J. (1989). Cómo pensamos. Nueva exposición de la relación entre pensamiento y proceso educativo. Barcelona: Ediciones http://villaeducacion.mx/descargar.php?idtema=1341\&data=5605aa_como-pensamos.pdf

Díaz, F. y Hernández, G. (2010). Estrategias Docentes para un aprendizaje significativo. México: Mc Graw Hill.

Feldman, R. (2005). Psicología: con aplicaciones en países de habla hispana. (Sexta Edición) México: McGrawHill.

Hepp, P. (2008). El desafío de las TIC como instrumento de Aprendizaje. Ponencia del Seminario Internacional Cómo las TIC transforman la escuela. Buenos Aires: UNICEF. https://www.oei.es/historico/pdfs/las_tic_aula_agenda_politica.pdf

Hernández, R., Fernández, C., Baptista, P. (2014). Metodología de la Investigación. Sexta edición. México: McGrawHill.

Hernández, R., Orrego, R., Quiñones, S. (2018). Nuevas formas de aprender: La formación docente frente al uso de las TIC. Propósitos y Representaciones, 6(2), 671-701. http://www.scielo.org.pe/pdf/pyr/v6n2/a14v6n2.pdf 
Hurtado, J. (2015). El Proyecto de Investigación. Comprensión holística de la metodología y la investigación. Octava Edición. Venezuela: Ediciones Quirón.

Landazábal, D. (2011). Mediación en entornos virtuales de aprendizaje. Universidad del Bosque. Bogotá. Colombia. http://colombiaaprende.edu.co/html/mediateca/1607/articles106651_archivo.pdf

Lugo T., y Kelly V., (2011). La Gestión de las TIC en las escuelas: el desafío de gestionar la innovación. Ponencia del Seminario Internacional Cómo las TIC transforman la escuela. Buenos Aires:

UNICEF. https://www.oei.es/historico/pdfs/las_tic_aula_agenda_politica.pdf

Martelo, P. Caamaño, E. Gómez, V. Pinedo, J. y Castaño, W. (2019). Utilización de Herramientas Didácticas en el sector Educativo como factor clave para la Enseñanza. Aglala, 10(2), 255-266. https://doi.org/10.22519/22157360.1446

Ministerio de Educación Nacional de Colombia (MEN 2013). Competencias TIC para el desarrollo profesional docente. Colombia Aprende. http://aprende.colombiaaprende.edu.co/es/node/121202

Ministerio de Educación (2006). Estándares en Tecnología de la Información y la Comunicación para la Formación Inicial Docente. Santiago, Min educ. https://www.oei.es/historico/tic/Estandares.pdf

Morrissey, J. (2011). El uso de las TIC en la enseñanza y el Aprendizaje. Cuestiones y desafíos. Ponencia del Seminario Internacional Cómo las TIC transforman la escuela. Buenos Aires: UNICEF. https://www.oei.es/historico/pdfs/las_tic_aula_agenda_politica.pdf

Navarro, J. (2010). Aprendizaje y memoria humana. McGraw Hill. Madrid. España.

Organización para la Cooperación y el Desarrollo Económico OCDE (2010). Habilidades y competencias del siglo XXI para los aprendices del nuevo milenio en los países de la $O C D E$.

http://recursostic.educacion.es/blogs/europa/media/blogs/europa/informes/Habilidades_y_comp etencias_siglo21_OCDE.pdf

Organización de las Naciones Unidas para la Educación, la Ciencia y la Cultura UNESCO (2013). Estándares de competencias TIC para docentes. http://eduteka.icesi.edu.co/articulos/EstandaresDocentesUnesco 
Paz, A.; Sánchez, A.; Atencio, X.; Balbuena, A. y Molero M. (2017). Habilidades cognitivas y lingüísticas. Venezuela: Fondo editorial URBE.

Pérez, I., Builes, L., Rivera, A. (2017). Estrategias para implementar las TIC en el aula de clase como herramientas facilitadoras de la gestión pedagógica. Seminario: Uso de TIC y mejoramiento de la calidad educativa. Medellín, Colombia.

Pilonieta, G. (2011). Modificabilidad Estructural Cognitiva y Educación. Bogotá. Colombia: Editorial Magisterio.

https://www.cedd.net/redis/index.php/redis/article/view/330

Programa para la Evaluación Internacional de Alumnos PISA (2018). Resultados De PISA 2018. OECD, Volumen I-III.

Servicio Nacional de Aprendizaje SENA (2019). Informe de Gestión 2019. https://www.sena.edu.co/esco/transparencia/Lists/Informes\%20de\%20gesti\%C3\%Bn/info _gest_2019.pdf

Tobón S. (2013). Formación Integral y Competencias. Pensamiento complejo, currículo, didáctica y evaluación. $4^{\text {ta }}$. Edición. Bogotá, Colombia. ECOE. https://www.researchgate.net/profile/Sergio_Tobon4/publication/319310793_Formacion_ integral_y_competencias_Pensamiento_complejo_curriculo_didactica_y_evaluacion/link s/59a2edd9a6fdcc1a315f565d/Formacion-integral-y-competencias-Pensamientocomplejo-curriculo-didactica-y-evaluacion.pdf

Valencia T., Serna A., Ochoa S., Caicedo A., Montes J. y Chávez J., (2016). Competencias y estándares TIC desde la dimensión pedagógica; Una perspectiva desde los niveles de apropiación de las TIC en la práctica educativa docente. Pontificia Universidad Javeriana - Cali. Multimedios. Colombia.

http://www.unesco.org/new/fileadmin/MULTIMEDIA/FIELD/Santiago/pdf/Competencia s-estandares-TIC.pdf

Yanes, J. (2011). Las TIC y la crisis de la educación. Biblioteca digital virtual. https://virtualeduca.org/documentos/yanez.pdf 